\title{
Numerical Model for Cavitational Flow in Hydraulic Poppet Valves
}

\author{
Sandor I. Bernad ${ }^{1}$ and Romeo Susan-Resiga ${ }^{2}$ \\ ${ }^{1}$ Center of Advanced Research in Engineering Sciences, Romanian Academy, Timisoara Branch, 300223 Timisoara, Romania \\ ${ }^{2}$ Department of Hydraulic Machinery, Politehnica University of Timisoara, 300222 Timisoara, Romania
}

Correspondence should be addressed to Sandor I. Bernad, sbernad@mh.mec.upt.ro

Received 9 January 2012; Accepted 10 April 2012

Academic Editor: Guan Heng Yeoh

Copyright ( 12012 S. I. Bernad and R. Susan-Resiga. This is an open access article distributed under the Creative Commons Attribution License, which permits unrestricted use, distribution, and reproduction in any medium, provided the original work is properly cited.

\begin{abstract}
The paper presents a numerical simulation and analysis of the flow inside a poppet valve. First, the single-phase (liquid) flow is investigated, and an original model is introduced for quantitatively describing the vortex flow. Since an atmospheric outlet pressure produces large negative absolute pressure regions, a two-phase (cavitating) flow analysis is also performed. Both pressure and density distributions inside the cavity are presented, and a comparison with the liquid flow results is performed. It is found that if one defines the cavity radius such that up to this radius the pressure is no larger than the vaporization pressure, then both liquid and cavitating flow models predict the cavity extent. The current effort is based on the application of the recently developed full cavitation model that utilizes the modified Rayleigh-Plesset equations for bubble dynamics.
\end{abstract}

\section{Introduction}

In hydraulic power systems, cavitation most frequently occurs in system valves, pumps, and actuators. Large differences in pressure are a frequent cause of small-scale cavitation in chambers of four-way spool valves, while highfrequency motion of a valve-controlled actuator can lead to large-scale cavitation in the cylinder. Another source of cavitation in hydraulic power systems is the improper filling of the pistons on an axial-piston pump. Either during transient loading or under steady-state operation, cavitation can occur in the return chamber of directional control valves because of the large pressure drop across the orifice. It is of interest to know the potential cavitation damage, as well as any effect of cavitation on system performance under both steady and unsteady flow conditions.

The flow inside the poppet valve is a complex process that is strongly dependent on the details of the valve geometry, the fluid properties, and the operating conditions. Separation and reattachment of jets can have a profound effect on the flow pressure and force characteristics as well as influencing the susceptibility to cavitations.
Hydraulic valves differ from process control valves in application and design. Hydraulic valves are typically used for controlling pressures and, therefore, are of the quick opening type of characteristics. Quick opening valves utilize plugs shaped in the form of a truncated cone with relatively large clearances between the plug and the seat. Or sometimes these valves utilize a disc for a poppet plug. Process control valves on the other hand are used for precise control of the fluid flow rate due to the linear or equal percentage characteristics. These type of valves usually have small clearances between the plug and the seat. Despite these differences, many of the flow phenomena in the hydraulic valve such as recirculation and jet separation and reattachment also occur in the process control valve.

A poppet valve is a seating-type valve. For this kind of valve the moving element (the poppet), usually can have a spherical or conical shape. Always, the poppet moves in a perpendicular direction to the seat. Because of the several advantages that are associated with poppet valves such as ease of manufacture, minimum leakage, and insensibility to clogging by dirt particles, poppets have been used for as pressure regulators and relief valves. The operation 
of this type of valve is quite simple. The fluid pressure counterbalances the spring force and allows fluid escape through the annular passage way between the poppet and the seat.

Separating interior flows are of the utmost importance for the performance of wide variety of technical applications [1-5]. Many industrial designs today have to compromise between the hydrodynamical function and other competing functions, for example, size or mechanical function. In such compromised designs undesired separation is more likely to occur, this drastically decreases the performance of the design. In such cases, active or passive devices that increase their near-wall momentum can be used to remove or reduce the separation $[2,3,6]$.

The presence of flow separation in the valve passage and the occurrence of different flow patterns have previously been identified in a number of investigations of different valve geometries. In the extensive work carried out by Tanaka in 1929 [7], it was observed that discontinuities in the flow occurred when investigating the flow quantity across the valve for different valve lifts.

The experimental work of hydraulic valves extends back over many years. Johnston et al. [8] studied forces on the valve plug as well as the pressure-flow characteristics for several different plug and seat arrangements. Schrenk [9] published work on the pressure-flow characteristics of poppet and disk valves. Stone [10] studied the characteristics of poppet valves with sharp-edged seats, small openings, and low Reynolds number. McCloy and McGuigan [11] studied the effects of the downstream chamber size in a two-dimensional model of a poppet. Some researchers have attempted to analytically predict flow through poppet valves. Von Mises [5] predicted the contraction coefficient for flow through an orifice using potential flow. Fluid forces on the plug are often estimated using simple concepts of fluid momentum change through the valve $[3,10]$. Recently CFD has been combined with experimental work to analyze hydraulic valves. Vaughan and Johnston [12] modeled the valve reported experimentally by Johnston et al. [8].

Weclas et al. [13] presented a comprehensive investigation into flow separation in the inlet valve passage using measurement techniques such as discharge coefficient measurements, surface flow visualization using an oil streak technique, and detailed flow measurements using LDA. The detailed flow measurements at the valve exit plane and the surface flow visualization showed the flow separation in the valve passage and identified its distribution around the valve periphery for generic inlet port geometries.

A successful design of poppet valves requires a through analysis of both velocity and pressure fields, with the aim of improving the poppet/seat geometry. Technological considerations lead to sharp corners, which in conjunction with very narrow passages produce regions of extremely high gradients in the flow field. As pointed in [14-16], the poppet flow is not easily suited to classical mathematical analysis.

The relative simple geometry (Figure 1), produces a very complicated viscous flow field (Figure 2), which can be realistically investigated only by using computational fluid mechanics. Powerful numerical tools, such as FLUENT

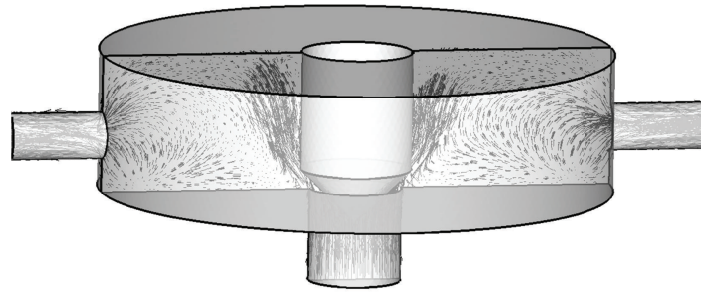

FIGURE 1: Velocity vector field and streamlines in meridional section plane.

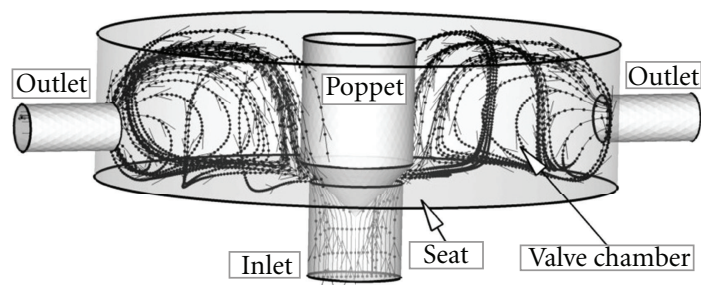

FIGURE 2: Flow field representation using selected streamlines inside the poppet valve.

software [17], are now available for investigating flows through arbitrary geometries.

Figure 1 shows the velocity vector field in meridional section plane overlaid with selected streamlines in the $3 \mathrm{D}$ computational domain. Velocity vector field in section plane shows the large recirculation region confirming the very strong nature of the helical vortex flow in this poppet valve geometry (Figure 2). The vortex evolution is clearly influenced by the position of the valve outlet section position.

As shown in our previous work [18], the cavitation region is relatively large in a poppet valve chamber; thus we expect a significant change of the flow field compared with the single-phase flow. One of the main goals of this paper is to explore the cavitating flow by using a two-phase flow model.

Section 2 presents a simple cavitating flow model employed by the commercial code FLUENT, and results obtained by using this model are presented in Section 5 . The main question addressed in this paper is whether or not the single-phase flow simulation correctly predicts the vapor cavity radius and location. In addition, we also examine the differences between single-phase and two-phase models in terms of the flow rate and streamline pattern.

\section{Cavitating Flow Modeling}

Cavitating flows are very sensitive to the formation and transport of vapour bubbles, the turbulent fluctuations of pressure and velocity, and the magnitude of noncondensable gases, which are dissolved or ingested in the operating liquid $[5,10]$.

Numerical simulation of two-phase cavitating flows is an ongoing research effort with the ambitious goal to compute the unsteady evolution for cavities growth and collapse. The CFD community has developed so far a set of mature 
techniques for simulating single-phase viscous flows, and the past half century of accumulated experience may very well serve to shape the numerical cavitating flow research. Early studies rely on the potential flow theory [19]. This approach is now able to correctly describe partially cavitating twodimensional hydrofoils, including the reentrant jet cavity closure model [20]. However, extension to 3D problems and other types of cavitating flows seems to be out of reach for the potential flow model.

Although basic cavitation theoretical studies deal with bubble (or bubble clouds) dynamics by solving for the vapour-liquid interface, most of the practical cavitating flows are approached using a homogeneous flow theory. The main idea is to consider a single variable density fluid, without explicit phase interfaces. This model has emerged after carefully examining available experimental investigations, as well as by evaluating the computational costs involved in cavitating flows modelling. A review of cavitating flows numerical studies over the past decade can be found in [21], where various Reynolds Averaged Navier-Stokes (RANS) solvers have been modified to account for the secondaryphase (vapour and gas) dynamics.

The mixture model is used in the current work for the numerical simulation of cavitating flows with the FLUENT expert code [17]. In this model, the flow is assumed to be in thermal and dynamic equilibrium at the interface where the flow velocity is assumed to be continuous.

The mixture is a hypothetical fluid with variable density,

$$
\rho_{m}=\alpha \rho_{v}+(1-\alpha) \rho_{l},
$$

ranging from liquid density for $\alpha=0$ to vapour density $\rho_{v}$ for $\alpha=1$. The vapour volume fraction,

$$
\alpha=\frac{\mathrm{Vol}_{\text {vapor }}}{\text { Vol liquid }_{\text {li }}+\text { Vol }_{\text {vapor }}},
$$

is an additional unknown of the problem. The mixture will of course satisfy the continuity equation

$$
\frac{d \rho_{m}}{d t}+\rho_{m} \nabla \cdot u_{m}=0
$$

where $d / d t$ denotes the material derivative. Next, one has to consider a momentum equation for the mixture. A simple choice would be to neglect the viscous effects and use the Euler equation. The system of equations can be then closed with a density-pressure relationship (equation of state). This approach can take advantage of a reach legacy of inviscid compressible solvers [22]. However, when considering a barotropic mixture, that is, the density depends solely on the pressure, some physics is lost. This can be easily seen when writing the vorticity transport equation

$$
\frac{\partial \boldsymbol{\omega}}{\partial t}+\mathbf{u} \cdot \nabla \boldsymbol{\omega}=\boldsymbol{\omega} \cdot \nabla \mathbf{u}+\frac{1}{\rho^{2}} \nabla \rho \times \nabla p+\text { viscous terms. }
$$

The second term in the right-hand side, which accounts for the baroclinic vorticity generation, vanishes when $\rho=$ $\rho(p)$. As a result, an important vorticity source is lost, especially in the cavity closure region [23].
Practical computations of industrial flows are using RANS equations with various turbulence modelling capabilities. This approach is embedded in most commercial codes currently available, for example, FLUENT [17]. As a result, it seems natural to build a cavitating flow model on top of such computational infrastructure.

An alternative to the equation of state is to derive a transport equation for the vapour volume fraction. The continuity equation (3), together with (1), gives the velocity divergence as

$$
\nabla \cdot u_{m}=-\frac{1}{\rho_{m}} \frac{d \rho_{m}}{d t}=\frac{\rho_{l}-\rho_{v}}{\rho_{m}} \frac{d \alpha}{d t} .
$$

Using (5), the conservative form of the transport equation for $\alpha$ can be easily written:

$$
\frac{\partial \alpha}{\partial t}+\nabla \cdot\left(\alpha u_{m}\right)=\frac{1}{\rho_{v}}\left[\frac{\rho_{v} \rho_{l}}{\rho_{m}} \frac{d \alpha}{d t}\right] .
$$

Equation (6) can be also written for the liquid volume fraction, $1-\alpha$ :

$$
\frac{\partial(1-\alpha)}{\partial t}+\nabla \cdot\left[(1-\alpha) u_{m}\right]=\frac{1}{\rho_{l}}\left[-\frac{\rho_{v} \rho_{l}}{\rho_{m}} \frac{d \alpha}{d t}\right] .
$$

The factor in square brackets in the r.h.s. of (6) and (7) is the interphase mass flow rate per unit volume:

$$
\dot{m}=\frac{\rho_{v} \rho_{l}}{\rho_{m}} \frac{d \alpha}{d t} .
$$

If we add term by term (6) and (7), we end up with an inhomogeneous continuity equation of the form

$$
\nabla \cdot u_{m}=\dot{m}\left(\frac{1}{\rho_{v}}-\frac{1}{\rho_{l}}\right),
$$

which is used in [24] to replace the homogeneous equation (3).

Finally, the vapour volume fraction transport equation is written as

$$
\frac{\partial \alpha}{\partial t}+\nabla \cdot\left(\alpha u_{m}\right)=\frac{1}{\rho_{v}} \dot{m} .
$$

This is the equation for the additional variable $\alpha$ to be solved together with the continuity and momentum equations.

Most of the efforts in cavitation modelling are focused on correctly evaluating $\dot{m}$. One approach has been proposed by Merkle et al. [25], by modelling the phase transition process similar to the chemically reacting flows. This model was successfully employed by Kunz et al. [26] in a variety of cavitating flows. However, the model constants are chosen somehow arbitrary, and this choice ranges several orders of magnitude from one problem to another. Senocak and Shyy attempt a derivation of an empiricism-free cavitation model [23] in order to avoid the evaporation/condensation parameters introduced by Merkle. 
A different approach is proposed by Schnerr and Sauer [27], who consider the vapour-liquid mixture as containing a large number of spherical bubbles. As a result, the vapour volume fraction can be written as

$$
\alpha=\frac{n_{b}(4 / 3) \pi R^{3}}{1+n_{b}(4 / 3) \pi R^{3}},
$$

where the number of bubbles per volume of liquid, $n_{b}$, is a parameter of the model.

From (11) we can easily get

$$
\frac{d \alpha}{d t}=\alpha(1-\alpha) \frac{3 \dot{R}}{R}
$$

where $\dot{R}$ is the bubble vapour-liquid interface velocity. A simplified Rayleigh equation can be used to compute

$$
\dot{R} \equiv \frac{d R}{d t}=\operatorname{sgn}\left(p_{v}-p\right) \sqrt{\frac{2}{3} \frac{\left|p_{v}-p\right|}{\rho_{l}}} .
$$

Of course the bubble grows if the mixture pressure is less than the vaporization pressure, $p<p_{v}$, and collapses when $p>p_{v}$. The bubble collapse, as modelled by the Rayleigh second-order differential equation, is much more rapid than the bubble growth. However, the above model seems to make no such difference between growth and collapse.

The present paper employs the mixture model, as implemented in the FLUENT commercial code, with the cavitation model described by (8), (12), and (13).

Physically, the cavitation process is governed by thermodynamics and kinetics of the phase change process. The liquid-vapor conversion associated with the cavitation process is modeled through two terms, which represents, respectively, condensation and evaporation. The particular form of these phase transformation rates forms the basis of the cavitation model.

\section{The Numerical Approach}

To simulate the cavitating flow the numerical code FLUENT [17] was used. The code uses a control-volume-based technique to convert the governing equations in algebraic equations that can be solved numerically. This control volume technique consists of integrating the governing equations at each control volume, yielding discrete equations that conserve each quantity on a control-volume basis. The governing integral equations for the conservation of mass and momentum and (when appropriate) for energy and other scalars, such as turbulence and chemical species, are solved sequentially. With the governing equations being nonlinear (and coupled), several iterations of the solution loop must be performed before a converged solution is obtained. The flow solution procedure is the SIMPLE routine [17]. This solution method is designed for incompressible flows, thus being implicit. The full Navier-Stokes equations are solved. The flow was assumed to be steady, and isothermal. In these calculations turbulence effects were considered using turbulence models, as the $k-\varepsilon$ RNG models, with the modification of the turbulent viscosity for multiphase flow. To model the flow close to the wall, the standard wall-function approach was used, and then the enhanced wall-function approach has been used to model the near-wall region (i.e., laminar sublayer, buffer region, and fully-turbulent outer region). For this model, the used numerical scheme of the flow equations was the segregated implicit solver. For the model discretization, the SIMPLE scheme was employed for pressure-velocity coupling, second-order upwind for the momentum equations, and first-order upwind for other transport equations (e.g., vapor transport and turbulence modeling equations). Computational domain is discretized using the GAMBIT preprocessor [17]. The flow close to the body surface is of particular importance in the current study, the mesh structure in the computational domain deliberately reflects this concern by heavily clustering the mesh close to the solid surface of the body so that the boundary layer mesh is used enclosing the body surface.

\section{Validation of the Cavitating Flow Model}

Before any attempt of computing cavitating hydrofoil flows, we have tested the model described in Section 2 on a benchmark problem. In a previous paper [28] we have computed the cavitating flow over a blunt body, where our numerical solutions are in a good agreement with experimental data obtained by Rouse [29]. In this paper we analyse the case of a hemispherical cavitator. The flow with and without cavitation computed for the axi-symmetric cavitator with blunt fore-body and numerical results is compared with experimental data. For this particular axisymmetric body, Rouse and McNown [29] have provided the pressure coefficient distribution along the body.

In this paper we used the volume of fluid method (since it is essentially based on the transport of the local volume fraction of the liquid) implemented in FLUENT code to capture the interface between fluid and vapour.

The volume of fluid methods belong to the group of Eulerian techniques. The basic idea of the VOF method is to consider a color function, defined as the volume fraction of one of the fluids within each cell, to capture the interface. This function will be unity if the cell is filled with the gas phase, zero if the cell is filled with the liquid phase, and between zero and one if the cell contains the interface. Interfaces are then generally reconstructed using a piecewise linear interpolation. The main strength of the volume of fluid method is that it conserves mass exactly as it works directly with the volume fraction. In general, a VOF algorithm solves the problem of updating the volume fraction field given the fixed grid, the velocity field, and the volume fraction field at the previous step.

Figure 4 shows the distribution of $\alpha$ around a hemispherical fore-body, for a cavitation number

$$
\sigma=\frac{p_{\infty}-p_{v}}{(1 / 2) \rho_{l} U_{\infty}^{2}}=0.3
$$

Most of the computational domain constrains only liquid, $\alpha=0$, but within the region with $p<p_{v}$ the vapour phase is formed with $0<\alpha<1$. 


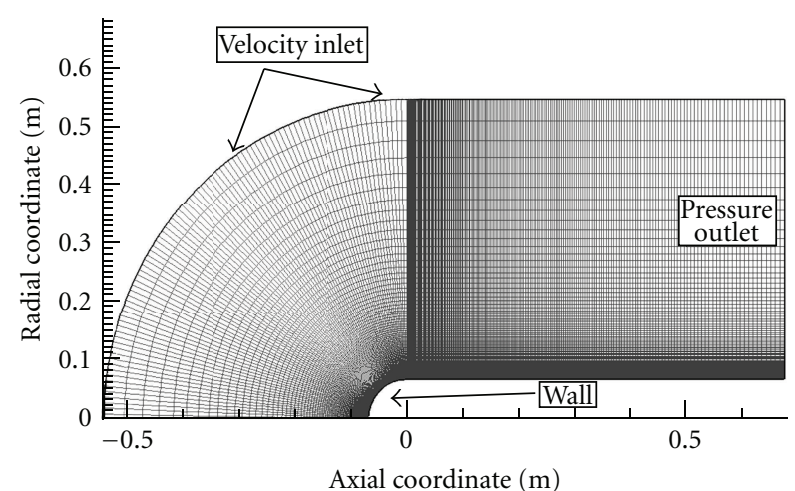

Figure 3: Computational domain and boundary conditions for hemispherical fore-body.

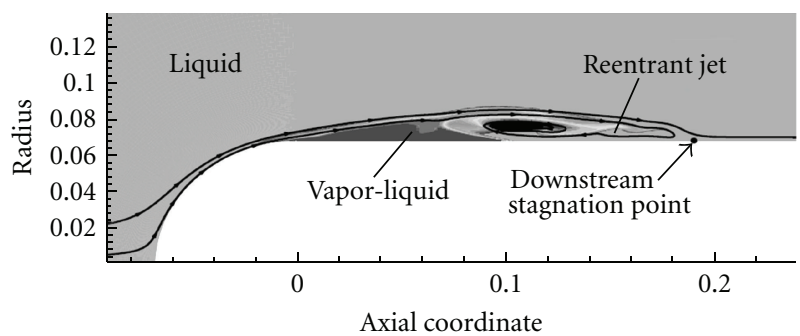

FIGURE 4: Vapour volume fraction and selected streamlines for cavitating flow around a hemispherical fore-body for $\sigma=0.3$.

Cavitation occurs as a result of the flow acceleration over the body surface resulting in regions with pressures lower than the vapor pressure (Figures 3 and 4). Then, the water transforms to vapor in these regions, thereby forming vapor-filled cavities. These cavities collapse when the local pressure becomes larger than the vapor pressure, with a reentrant water jet, and the flow generally becomes unsteady. Thus, an irregular cyclic process of bubble formation and growth occurs, followed by the filling and finally breaking off of the bubble. Due to cavitation, large-density and viscosity gradients arise at the interfaces between nearly incompressible fluids.

Within the cavity there are regions practically filled with gas (the first half) and regions with a gas-liquid mixture corresponding to the reentrant jet dispersion and vaporisation. The present simulation considers a steady flow, corresponding to a stable attached cavitation. However, when the reentrant jet crosses the cavity boundary, a large part of the cavity detaches and is transported downstream, while the remaining part starts growing again.

The qualitative analysis above is completed with a quantitative comparison shown in Figure 5. The pressure coefficient is plotted against the dimensionless curvilinear abscissa along the body, originating at the axis,

$$
c_{p}=\frac{p-p_{\infty}}{(1 / 2) \rho_{l} U_{\infty}^{2}} .
$$

The numerical results correspond to the dashed line and agree very well with the experimental data [29]. In

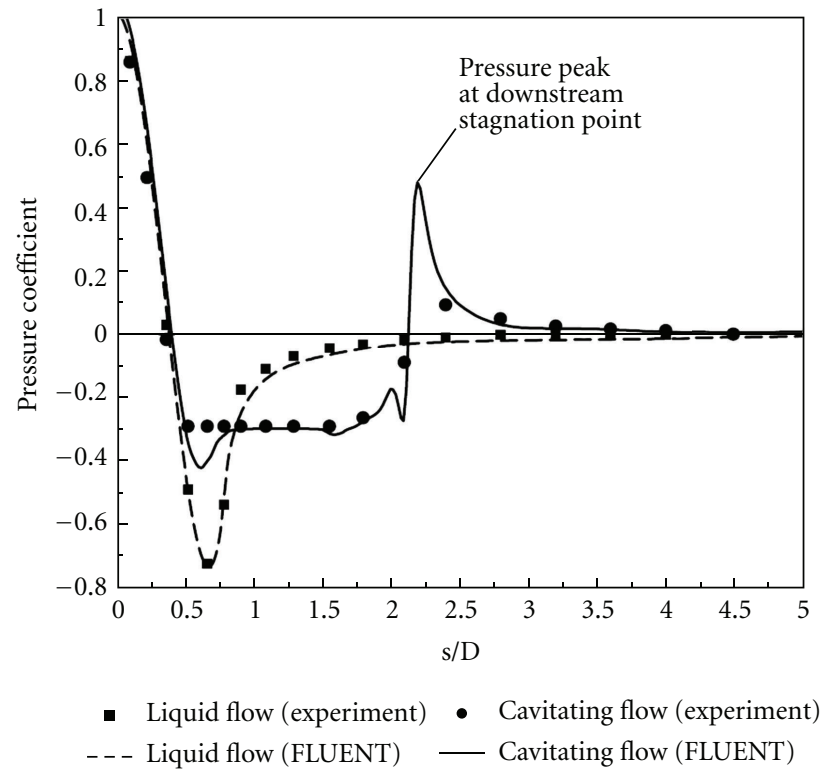

Figure 5: Pressure coefficient distribution on the hemispherical fore-body. The curves correspond to the present computations, while the points are experimental data of Rouse and McNown [29].

the cavitation model the vaporization pressure is adjusted to obtain the cavitation number $\sigma=0.3$. As a result, the negative values of pressure coefficient are limited within the cavity at $-\sigma$. However, at cavitation inception (upstream and of the cavity) the pressure coefficient still drops below $-\sigma$ as the bubbles are transported faster than they can grow. At the end of the cavity there is a sharp increase in $c_{p}$, corresponding to the exact location of the downstream stagnation point from Figure 5.

\section{Cavitating Flow Simulation and Analysis}

Flow inside the poppet valve has been investigated considering a typical computational domain presented in Figure 6(a). Pressure conditions were considered both on the inlet and outlet sections of the axisymmetric domain, and the rest of the boundary is considered a solid wall (Figure 6(b)). In order to perform a quantitative analysis of the complicated streamline pattern inside the poppet valve chamber, an original theoretical vortex model has been proposed [16], thus allowing a parametric study of the poppet valve flow evolution in the whole range of poppet displacement.

A typical streamline pattern for the liquid flow through the poppet valve is presented in Figure 7. Three main vortices are developed in the poppet valve chamber. The first two vortices, V1 and V2, are rotating counterclockwise and clockwise, respectively, and are generated on the lefthand side and right-hand side, respectively, of the liquid jet issued from the poppet-seat opening. The third vortex, V3, is generated beyond the valve chamber, in the outflow channel. Such a qualitative analysis of the flow field has been performed also by Dietze [15], who used flow visualization to validate the numerical results, that is, the streamline pattern. 


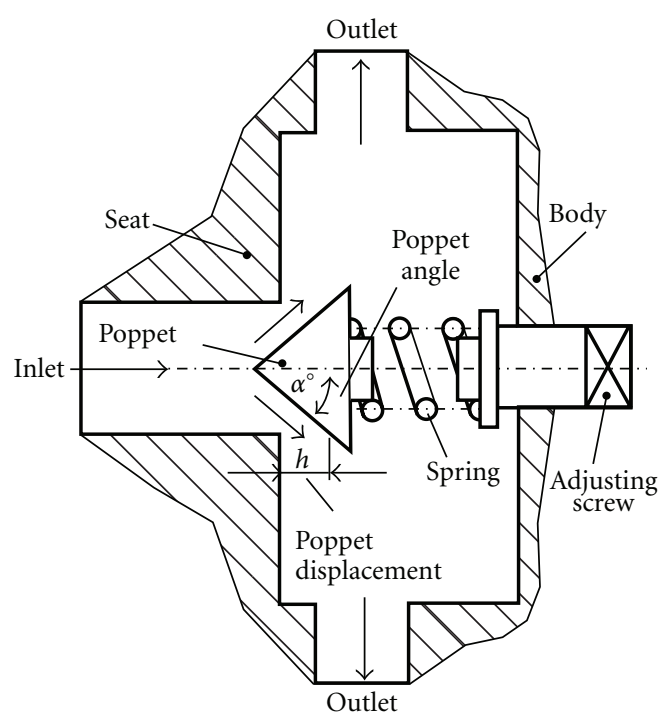

(a)

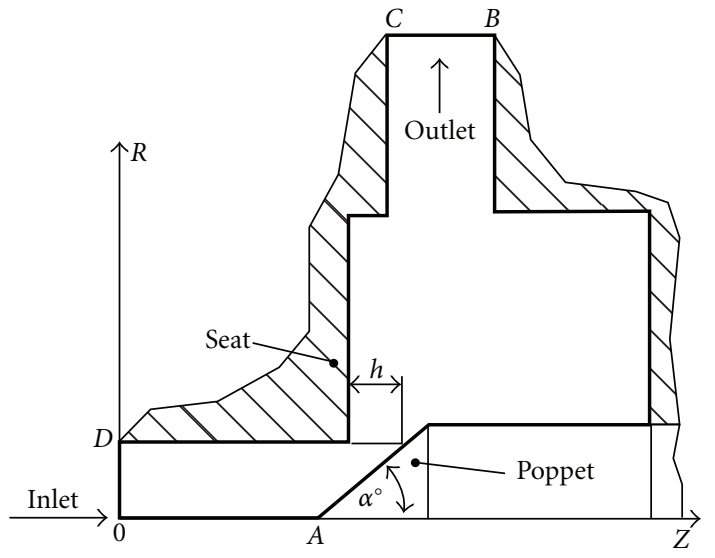

(b)

FIGURE 6: Cross-sectional view of a typical poppet valve and the corresponding computational domain in a meridian half-plane.

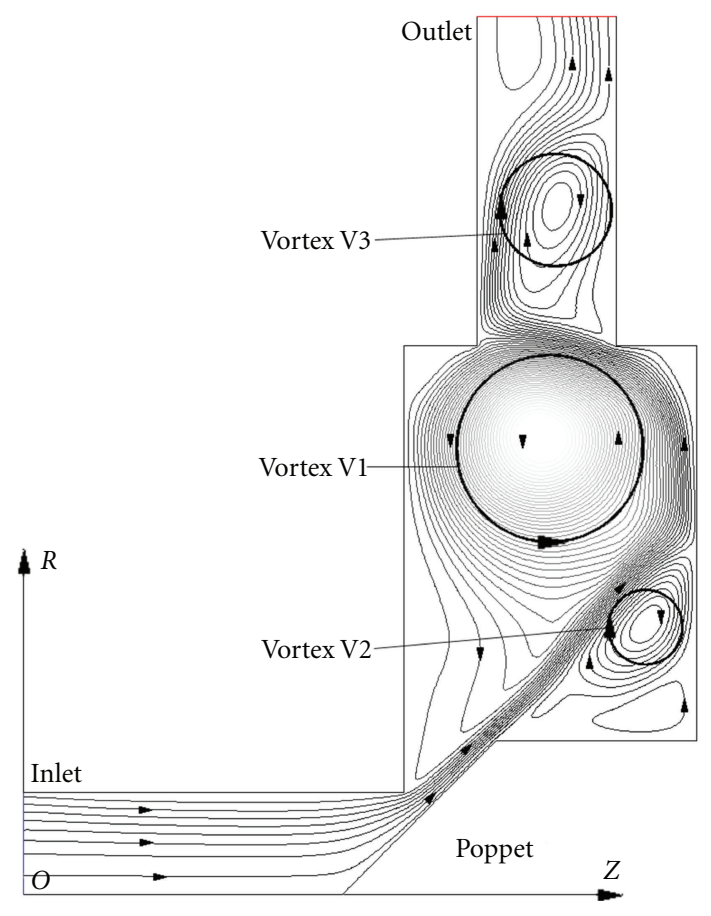

Figure 7: Streamline pattern in the meridian half-plane for a poppet valve with $28 \mathrm{~mm}$ inlet diameter, $45^{\circ}$ poppet cone angle, and $5,6 \mathrm{~mm}$ poppet displacement, operating at inlet/outlet pressure of 61 bar/1 bar.

However, Dietze does not provide a quantitative description of the velocity and pressure fields details.

In order to carry out the mesh sensitivity analysis, numerical simulations were carried out by varying the number of mesh elements in the computational domain. Initially, the domain was discretized into 26,500 elements.
The accuracy of the simulation results was then improved by employing a finer mesh that contained 60,700 elements. This number was further increased to 67,850 but resulted in no significant improvement in the results. Thus to maintain a balance between the computational cost and the numerical accuracy, we concluded (based on the mesh independence test) that the appropriate number of elements for our study is 41,970 .

The presence of flow separation in the valve passage (Figure 8) and the occurrence of different flow patterns have previously been identified in a number of investigations of different valve geometries. Bulloungh and Chin [1] performed the static pressure measurements along the valve cone and seat wall for different poppet displacements. Visualization of the valve passage flow in a transparent model was investigated for different poppet valve configurations by Johnston et al. [8]. Dietze [15] in his Ph.D. thesis presented a comprehensive investigation into flow separation in the valve passage using measurement techniques and flow visualization to validate the numerical results, that is, the streamline pattern.

As mentioned in Section 3, after obtaining a steady single-phase (liquid) flow solution, the FLUENT code allows turning on the cavitation model. As a result, vapor formation is enabled where the absolute pressure is smaller than the vaporization pressure. In order to obtain correct results the so-called operating pressure $p_{\text {op }}$ must be set to zero (it is set to the atmospheric pressure by default); therefore the gauge pressure $p_{\text {gauge }}$ will equal the absolute pressure $p_{\text {abs }}$,

$$
p_{\text {abs }}=p_{\text {op }}+p_{\text {gauge }} \text {. }
$$

This is particularly important for obtaining only positive absolute pressure values.

As shown in Figure 9, when the cavitating flow model is used the pressure inside the cavity becomes constant 


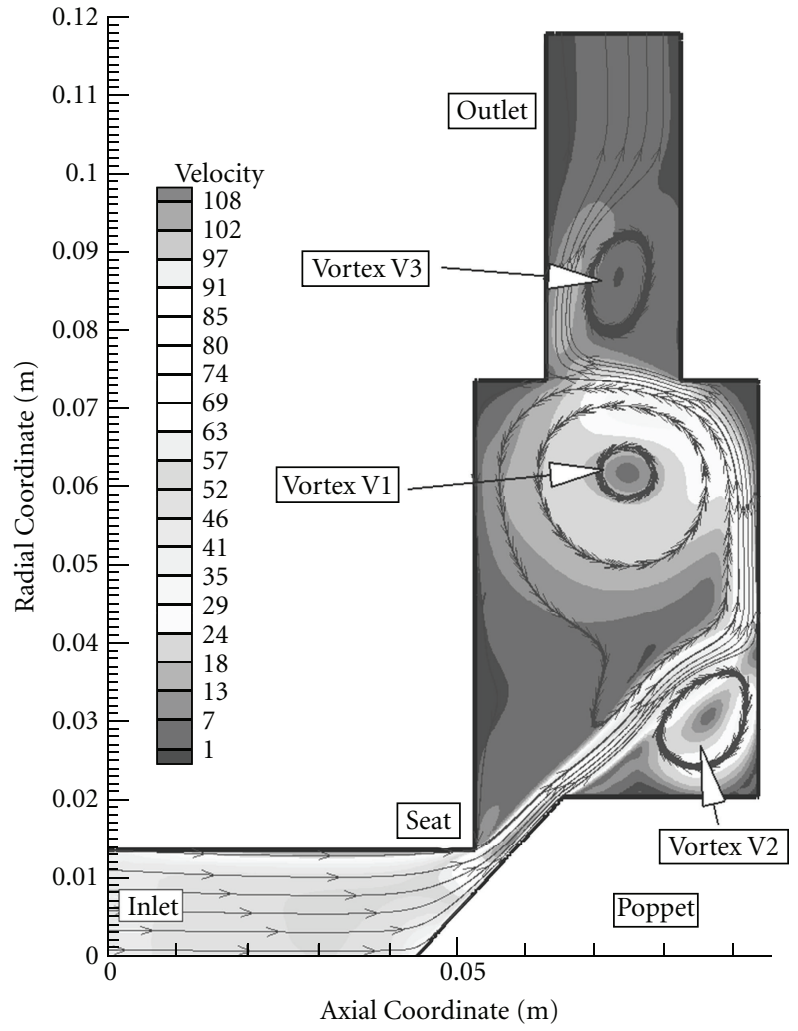

Figure 8: Streamline pattern and pressure field in the meridian half-plane of the poppet valve.

and equals the vaporization pressure, in concordance with cavitation physics. On the other hand, Figure 10 reveals that the pressure exceeds the vaporization pressure at the same radius of approximately $9 \mathrm{~mm}$ for both single-phase and two-phase models. However, one cannot say that the cavity radius is $9 \mathrm{~mm}$ since a continuous transition from vapor to liquid takes place.

If the cavity radius is defined such that inside the cavity the pressure does not exceed the vaporization pressure, then Figure 9 shows that both single-phase and cavitating flow models predict the same cavity extent. However, the above conclusion might be valid only for a particular value of the bubble density number $n$ (see (11)). As a result, we have performed the numerical simulation for three values for $n$, namely, $10^{4}, 10^{5}$, and $10^{6}$, within the range recommended by Kubota et al. [20]. The results are presented in Figure 10, with a zoom for the pressure range around zero. We can conclude that when a steady cavitating flow configuration is computed, the bubble density number has no significant influence on the results. This is particularly important since there is no need for additional information to choose the appropriate value for $n$.

Figure 11 shows the liquid-vapor mixture density, starting with the vapor region inside the cavity and ending with pure liquid. One can see that there is a large region containing a mixture of liquid and vapor, as it actually happens for industrial cavitation.

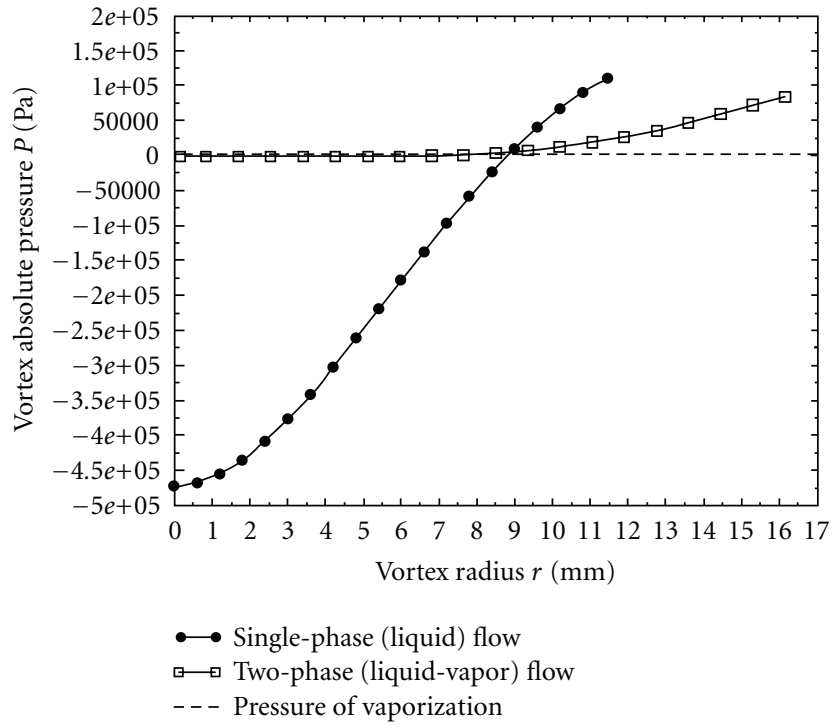

FIGURE 9: Radial pressure distribution inside the main vortex V1, computed for liquid flow (filled circles, see Figure 7) and liquidvapor flow (squares).

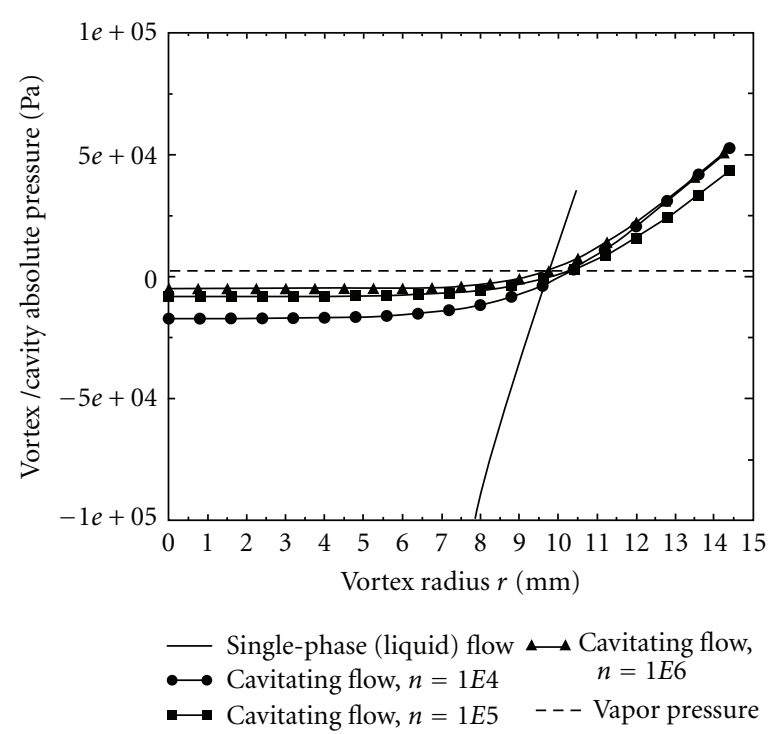

Figure 10: Influence of the bubble density number $n$ on the pressure distribution inside the cavity.

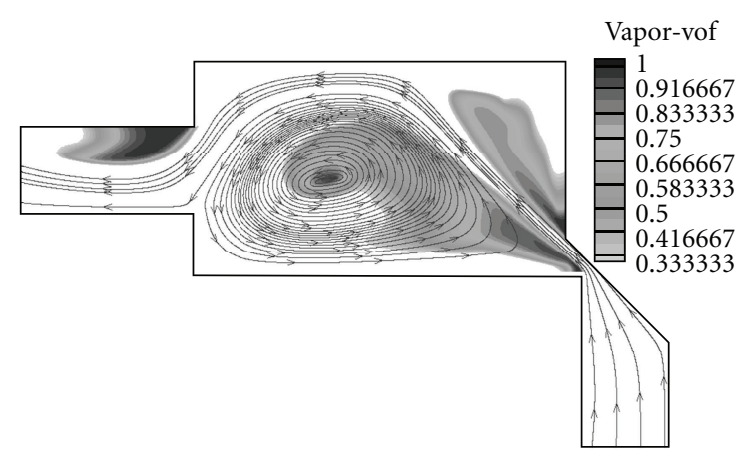

FIGURE 11: Radial distribution for the liquid-vapor mixture density inside the main vortex (cavity). 


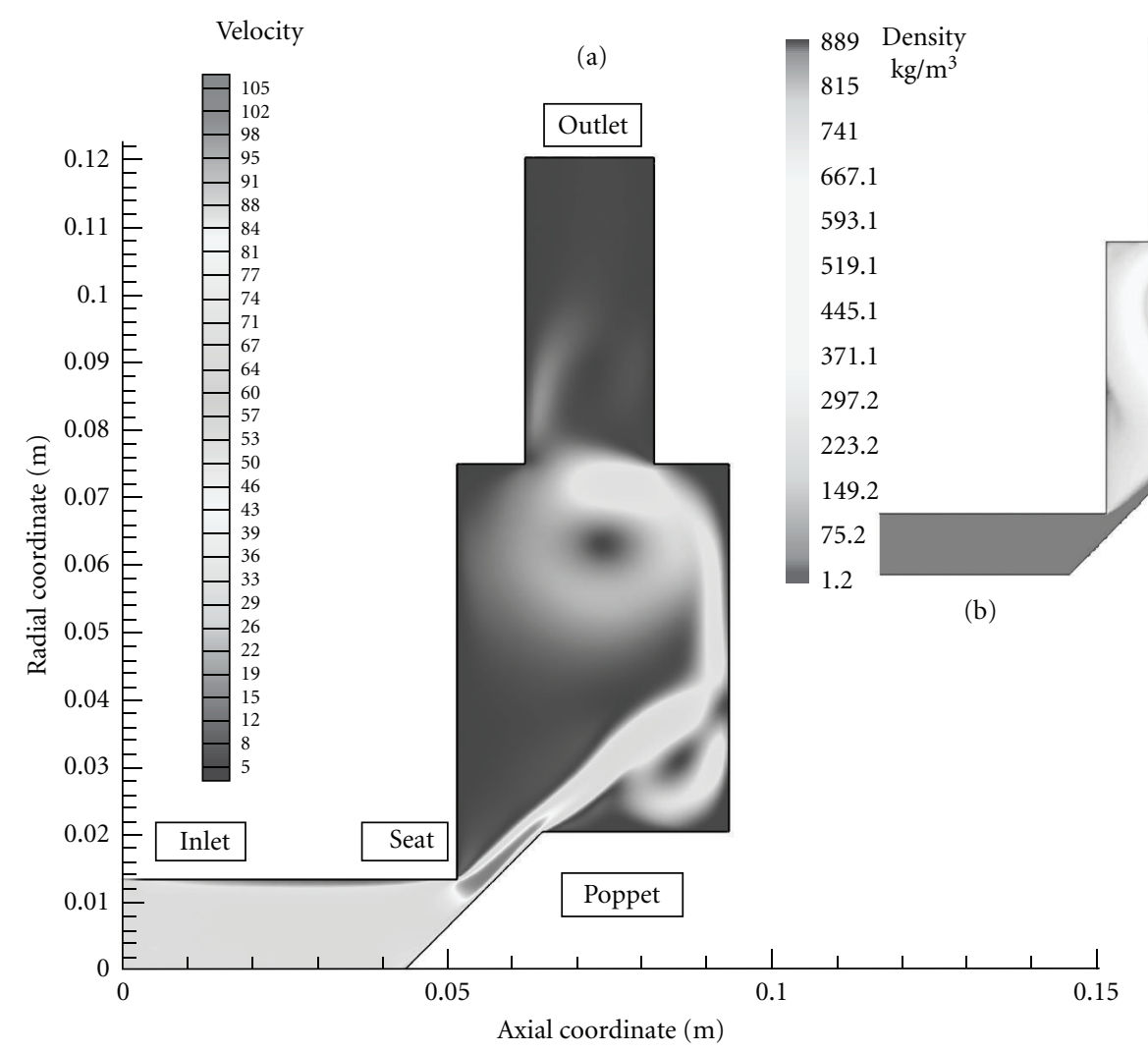

FIGURE 12: Velocity field and corresponding liquid-vapor mixture density distribution for cavitating flow.

A control valve creates a pressure drop in the fluid as it controls the flow rate. The profile of the fluid pressure, as it flows through the valve, is shown in Figure 11. The fluid accelerates as it takes a pressure drop through the valve trim. It reaches its highest velocity just past the throttle point, at a point called the vena contracta. The fluid is at its lowest pressure and highest velocity at the vena contracta. Past the vena contracta the fluid decelerates and some of the pressure drop is recovered as the pressure increases. The pressure in the vena contracta is not of importance until it is lower than the fluid vapor pressure. Then the fluid will quickly form vapor bubbles and, if the pressure increases above the vapor pressure, the vapor bubbles instantly collapse back to liquid (Figure 11).

The liquid flow rate will increase as the pressure drop increases. However, when cavitation vapor bubbles form in the vena contracta, the vapor bubbles will increasingly restrict the flow of liquid until the flow is fully choked with vapor. This condition is known as "choked flow" or "critical flow." When the flow is fully choked, the flow rate does not increase when the pressure drop is increased.

Cavitation will begin at the point of "incipient cavitation" and increase in intensity to the point of choked flow. Cavitation at point of "incipient cavitation" is not damaging and is almost undetectable. At some point between incipient and choked, the cavitation may damage most trim styles. The location of the "damage" point varies with trim style and material.
The radial distribution of the density inside the main vortex is presented in Figure 12. One can say that the vapourfilled cavity has a radius of $5 \mathrm{~mm}$, but since we have a smooth transition from the vapor region to the liquid region other conventional cavity radii might be defined.

The cavitating flow streamline pattern is not significantly altered in comparison with the one presented in Figure 7. However, one can notice that the main vortex is slightly shifted toward the axis of symmetry and the secondary vortex $\mathrm{V} 2$ becomes smaller.

Figure 12 shows the liquid-vapor mixture density, starting with the vapor region inside the cavity and ending with pure liquid. One can see that there is a large region containing a mixture of liquid and vapor, as it actually happens for industrial cavitation [30]. The radial distribution of the density inside the main vortex is presented in Figure 13. One can say that the vapour-filled cavity has a radius of $5 \mathrm{~mm}$, but since we have a smooth transition from the vapor region to the liquid region the above definition for the cavity radius in terms of pressure seems to be more appropriate.

As far as the flow rate is concerned, the liquid flow value is practically the same for cavitating flows. Further investigations are needed to elucidate this issue.

\section{Conclusions}

The paper presents a numerical investigation of cavitating flows using the mixture model implemented in the FLUENT 


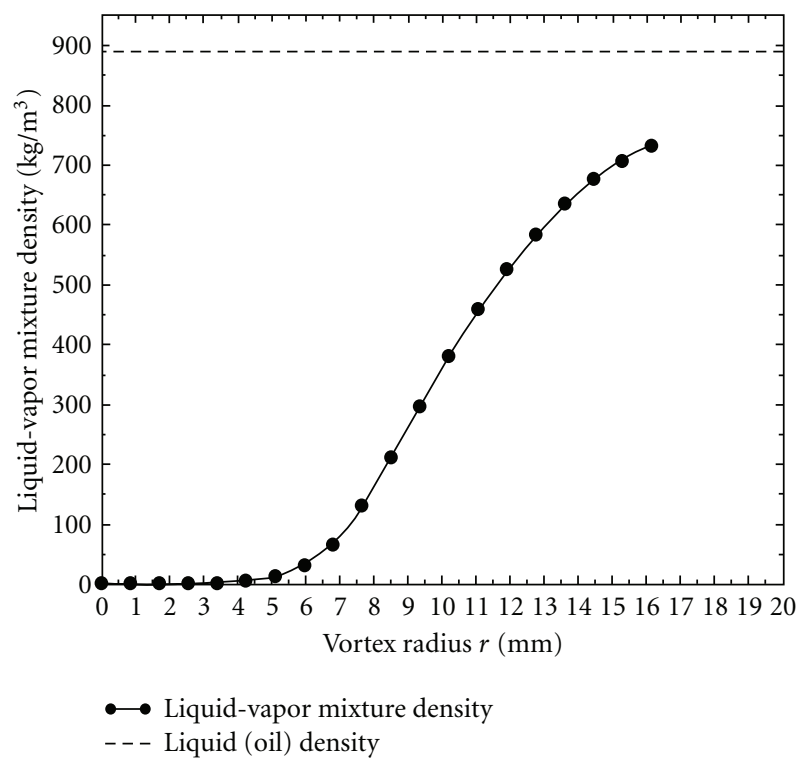

FIGURE 13: Radial distribution for the liquid-vapor mixture density inside the main vortex (cavity).

commercial code. The interphase mass flow rate is modelled with a simplified Rayleigh equation applied to bubbles uniformly distributed in computing cells, resulting in an expression for the interphase mass transfer. This is the source term for the vapor phase transport equation. As a result, the density of the liquid-vapor mixture is allowed to vary from the vapor density up to the liquid density.

The cavitation model is validated for the flow around a hemispherical body cavitator. The numerical results agree very well both qualitatively and quantitatively with the experiments. As a result we include that the present cavitational model is able to capture the major dynamics of the cavitating flows inside the hydraulic power equipment.

Cavitation damage problems are more likely to occur with water flow as water has a well-defined vapor pressure and the vapor bubble collapse is instantaneous. Hydrocarbon fluids have a less precise vapor pressure and are often a compound with several vapor pressures [31,32]. Cavitation damage with hydrocarbon fluids is usually less severe than water, as the bubble collapse is not as sudden and can be cushioned by other vapors. However, the vibration and flow noise problems remain $[3,10]$.

The fluid inlet pressure is proportional to the amount of energy available to cause cavitation damage. Higher inlet pressures will produce more intense and more damaging cavitation.

The generation and implosion of the vapor bubbles will cause vibration to the valve poppet that may cause wear between the poppet and the seat.

The generation and implosion of the vapor bubbles will cause significantly elevated flow noise in addition to vibration. The cavitation bubbles will form a vapor plume in the liquid. The larger the plume, the noisier the flow and the more likely it is to cause erosion damage. The size of the plume is dependent on trim style and severity of cavitation.
The cavitating flow pressure distribution displays a constant value inside the vapor filled cavity, with a slow increase as the mixture density increases. The investigation of the liquid-gas mixture density shows a smooth and continuous transition from vapor to liquid. This transition takes place over a relatively large region. The results obtained for a steady cavitating flow does not depend on the bubble number density. This conclusion might not be true when time-dependent evolutions are investigated.

As the authors proceed with this research, they focus on several areas including: (1) improved physical model for mass transfer and (2) extended application and validation for steady two-dimensional flows.

\section{Nomenclature}

$\begin{array}{ll}h: & \text { Poppet displacement }[\mathrm{m}] \\ n_{b}: & \text { Number of bubbles per unit volume of liquid } \\ & {\left[1 / \mathrm{m}^{3}\right]} \\ p_{0} & \text { Mixture pressure }[\mathrm{Pa}] \\ p_{v}: & \text { Vaporization pressure }[\mathrm{Pa}] \\ p_{\text {abs }}: & \text { Absolute pressure }[\mathrm{Pa}] \\ p_{\text {gauge }}: & \text { Gauge pressure }[\mathrm{Pa}] \\ p_{\text {op }}: & \text { Operating pressure }[\mathrm{Pa}] \\ R: & \text { Bubble radius }[\mathrm{m}] \\ \dot{R}: & \text { Bubble vapor-liquid interface velocity }[\mathrm{m} / \mathrm{s}] \\ \alpha: & \text { Vapour volume fraction }[-] \\ \rho_{m}: & \text { Liquid-vapor mixture density }\left[\mathrm{kg} / \mathrm{m}^{3}\right] \\ \rho_{v}: & \text { Vapor volume fraction density }\left[\mathrm{kg} / \mathrm{m}^{3}\right] \\ \sigma: & \text { Cavitation number }[-] \\ u_{m}: & \text { Liquid-vapor mixture velocity }[\mathrm{m} / \mathrm{s}] \\ \mathbf{u}: & \text { Absolute velocity }[\mathrm{m} / \mathrm{s}] \\ \boldsymbol{\omega}=\nabla \times \mathbf{u}: & \text { Vorticity }[1 / \mathrm{s}] \\ t: & \text { Time }[\mathrm{s}]\end{array}$

\section{Acknowledgment}

This work has been supported by the Romanian Academy annual program.

\section{References}

[1] W. A. Bulloungh and S. B. Chin, "A numerical study of the effects of poppet valve geometry on its flow characteristics," in Proceedings of the 9th International Symposium on Transport Phenomena in Thermal-Fluids Engineering, pp. 579-584, Singapore, June 1996.

[2] J. A. Davis and M. Stewart, "Predicting globe control valve performance-part I: CFD modeling," Journal of Fluids Engineering, vol. 124, no. 3, pp. 772-777, 2002.

[3] H. L. Sorensen, "Numerical and experimental analyses of flow and flow force characteristics for hydraulic seat valves with difference in shape," in Proceedings of the Bath Workshop on Power Transmission \& Motion Control, University of Bath, 1999.

[4] A. Maier, T. H. Sheldrake, and D. Wilcock, "Geometric parameters influencing flow in an axisymmetric IC engine inlet port assembly: part I-valve flow characteristics," Journal of Fluids Engineering, vol. 122, no. 4, pp. 650-657, 2000. 
[5] R. von Mises, "The calculation of flow coefficient for nozzle and orifice," VDA, vol. 61, pp. 21-23, 1916.

[6] A. Maier, T. H. Sheldrake, and D. Wilcock, "Geometric parameters influencing flow in an axisymmetric IC engine inlet port assembly: part II-parametric variation of valve geometry," Journal of Fluids Engineering, vol. 122, no. 4, pp. 658-665, 2000.

[7] K. Tanaka, "Airflow through suction valve of conical seat," Aeronautical Research Institute Report, Tokyo Imperial University, part 1, p. 262, part 2, p. 361, 1929.

[8] D. N. Johnston, K. A. Edge, and N. D. Vaughan, "Experimental investigation of flow and force characteristics of hydraulic poppet and disc valves," Proceedings of the Institution of Mechanical Engineers A, vol. 205, no. 3, pp. 161-171, 1991.

[9] E. Schrenk, Disc Valves, Flow Patterns, Resistance, and Loading, vol. T547, BHRA Publications, 1957.

[10] J. A. Stone, "Discharge coefficients and steady state flow forces for hydraulic poppet valves," Transactions of the ASME D, vol. 82, no. 1, pp. 144-154, 1960.

[11] D. McCloy and R. H. McGuigan, "Some static and dynamic characteristics of poppet valves," Proceedings of the Institution of Mechanical Engineers, vol. 179, no. 3, pp. 199-213, 1964.

[12] N. D. Vaughan and D. N. Johnston, "Numerical simulation of fluid flow in poppet valves," Proceedings of the Institution of Mechanical Engineers, vol. 206, no. 23, pp. 119-127, 1991.

[13] M. Weclas, A. Melling, and F. Durst, "Flow separation in the inlet valve gap of piston engines," Progress in Energy and Combustion Science, vol. 24, no. 3, pp. 165-195, 1998.

[14] S. Bernad, R. Susan-Resiga, I. Anton, and V. Ancusa, "Vortex flow modeling inside the poppet valve chamber-part 2," in Proceedings of the Bath Workshop on Power Transmission \& Motion Control (PTMC '01), pp. 161-176, Bath, UK, September 2001.

[15] M. Dietze, Messung und Berechnung der Innenströmung in hydraulischen Sitzventilen, Ph.D. thesis, Technische Universität Darmstadt, Düsseldorf, Geramny, 1996.

[16] R. Susan-Resiga, S. I. Bernad, and I. Anton, "Vortex flow modeling inside the poppet valve chamber," in Proceedings of the 7th Scandinavian International Conference on Fluid Power (SICFP '01), Linkoping, Sweden, May 2001.

[17] FLUENT 6.3 User's Guide, Ansys Fluent, 2006.

[18] S. Bernad, R. Susan-Resiga, S. Muntean, and I. Anton, "Cavitation phenomena in hydraulic valves. Numerical modelling," Proceedings of the Romanian Academy A, vol. 8, no. 2, pp. 151160, 2007.

[19] C. S. Martin, H. Medlarz, D. C. Wiggert, and C. Brennen, "Cavitation inception in spool valves," Journal of Fluids Engineering, vol. 103, no. 4, pp. 564-576, 1981.

[20] A. Kubota, H. Kato, and H. Yamaguchi, "A new modelling of cavitating flows: a numerical study of unsteady cavitation on a hydrofoil section," Journal of Fluid Mechanics, vol. 240, pp. 59-96, 1992.

[21] G. Wang, I. Senocak, W. Shyy, T. Ikohagi, and S. Cao, "Dynamics of attached turbulent cavitating flows," Progress in Aerospace Sciences, vol. 37, no. 6, pp. 551-581, 2001.

[22] D. R. van der Heul, C. Vuik, and P. Wesseling, "Efficient computation of flow with cavitation by compressible pressure correction," in Proceedings of the European Congress on Computational Methods in Applied Sciences and Engineering (ECCOMAS '00), Barcelona, Spain, September 2000.

[23] I. Senocak and W. Shyy, "Evaluation of cavitation models for Navier-Stokes computations," in Proceedings of the 2002 ASME Fluids Engineering Division Summer Meeting, Paper FEDSM2002-31011, pp. 395-401, July 2002.
[24] J. L. Kueny, Cavitation Modeling, Lecture Series: Spacecraft Propulsion, Von Karman, Institute for Fluid Dynamics, 1993.

[25] C. L. Merkle, J. Z. Feng, and P. E. O. Buelow, "Computational modeling of the dynamics of sheet cavitation," in Proceedings of the 3rd International Symposium on Cavitation, pp. 307-311, 1998.

[26] R. F. Kunz, D. A. Boger, T. S. Chyczewski, D. R. Stinebring, and H. J. Gibeling, "Multi-phase CFD analysis of natural and ventilated cavitation about submerged bodies," Proceedings of the 3rd ASME/JSME Joint Fluids Engineering Conference (FEDSM '99), 1999.

[27] G. H. Schnerr and J. Sauer, "Physical and numerical modeling of unsteady cavitation dynamics," in Proceedings of the 4th International Conference on Multiphase Flow, New Orleans, La, USA, 2001.

[28] S. Bernad, S. Muntean, R. Resiga, and I. Anton, "Numerical analysis of the cavitating flows," Proceedings of the Romanian Academy A, vol. 7, no. 1, pp. 33-45, 2006.

[29] H. Rouse and J. S. McNown, Cavitation and Pressure Distribution, Head Forms at Zero Angle of Yaw, vol. 32 of Studies in Engineering Bulletin, State University of Iowa, 1948.

[30] R. E. A. Arndt, "Cavitation in fluid machinery and hydraulic structures," Annual Review of Fluid Mechanics, vol. 13, pp. 273-328, 1981.

[31] E. Urata, "Thrust of poppet valve," Bulletin of The Japan Society of Mechanical Engineers, vol. 12, no. 53, pp. 1099-1109, 1969.

[32] E. Urata, "Cavitation erosion in various fluids," in Proceedings of the Bath Workshop on Pover Transmission \& Motion Control, University of Bath, September 1999. 

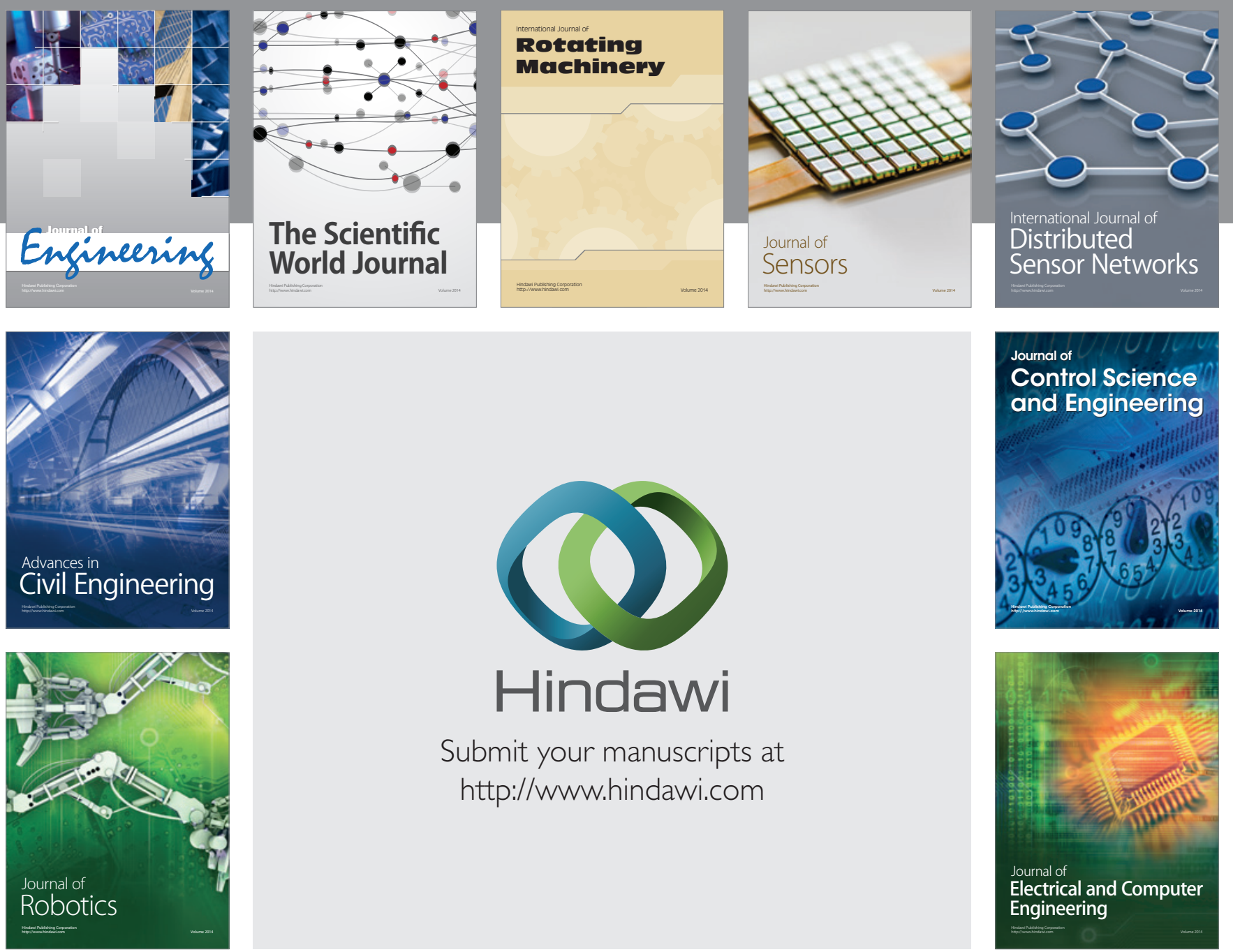

Submit your manuscripts at

http://www.hindawi.com
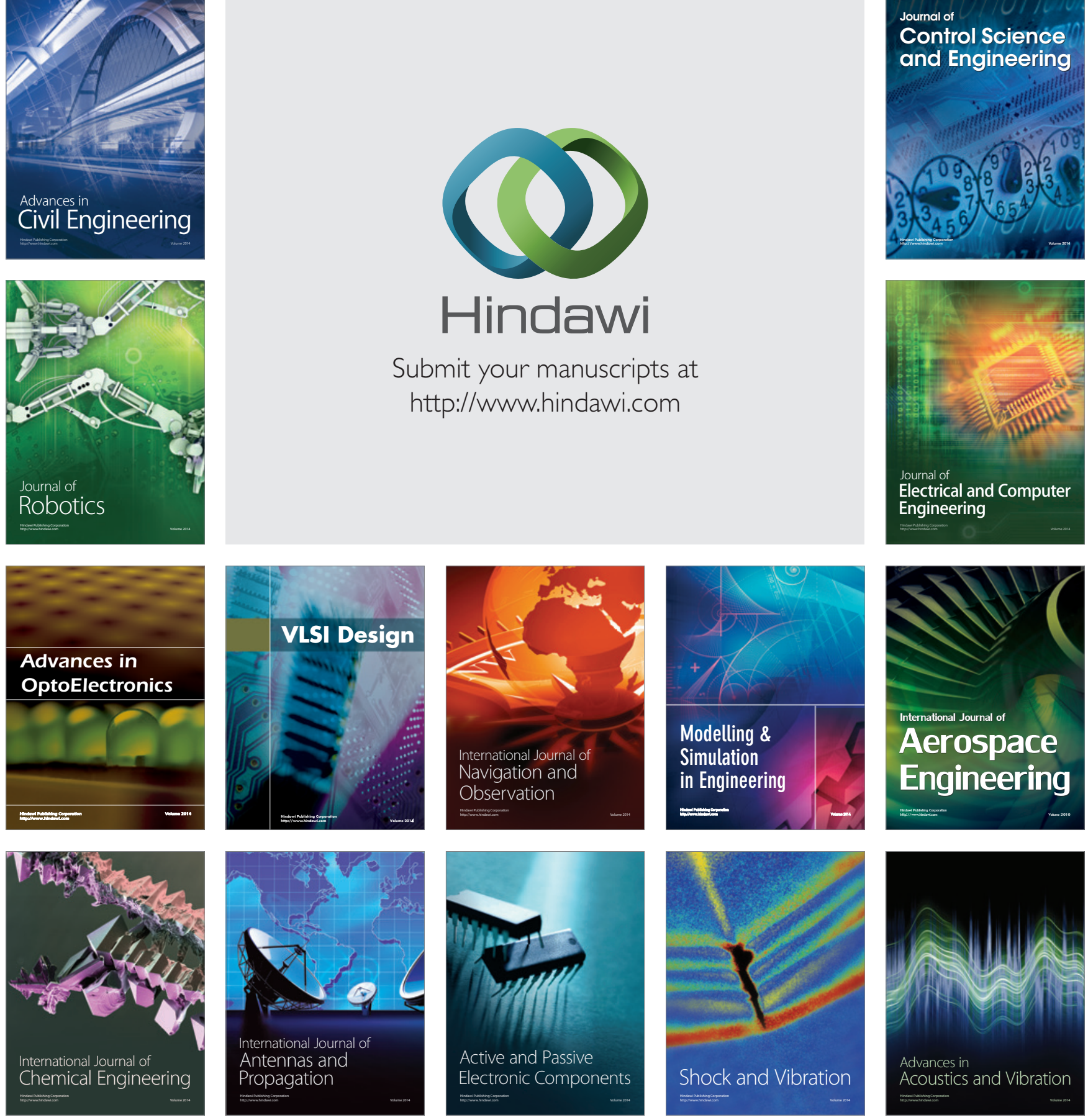